\title{
Cryo-SEM of hydrated high temperature proton exchange membranes
}

\author{
K. A. Perry, ${ }^{*}$ K.L. More, ${ }^{* *}$ L.R. Walker, ${ }^{* *}$ and B. C. Benicewicz*** \\ *Dept. of Chemistry and Chemical Biology, Rensselaer Polytechnic Institute, Troy, NY \\ **MST Division, Oak Ridge National Laboratory, Oak Ridge, TN \\ ***Dept. of Chemistry and Biochemistry \& USC NanoCenter, University of South Carolina, \\ Columbia, SC
}

Alternative energy technologies, such as high temperature fuel cells and hydrogen pumps, rely on proton exchange membranes (PEM).[1] A chemically and thermally stable PEM with rapid proton transport is sol-gel phosphoric acid (PA)-doped polybenzimidazole (PBI) membranes.[2] This research evaluates imaging techniques to characterize the gel structure of PA-doped PBI membranes.

Previous studies have shown that the gel structure of PA-doped PBI membranes is sensitive to preparation techniques that remove excess liquid for evaluation in the scanning electron microscope (SEM). SEM preparation techniques include water-exchange and dehydration by heat treatments. For example, when PA-doped PBI membranes underwent a water-exchange process to remove the acid, wide-angle X-ray scattering (WAXS) data showed structural rearrangement. It was determined that in order to accurately evaluate the microstructure of such membranes, the acid should be retained during imaging and analysis. A $120^{\circ} \mathrm{C}$ membrane heat treatment for 5 days to remove excess water was necessary to allow imaging in a high-resolution SEM (JEOL 6500) at room temperature (RT). WAXS data of the heat-treated membranes showed no structural rearrangement.

In order to elucidate the gel-structure of high temperature PEMs using SEM, heat-treated PA-doped para-PBI membranes were imaged and analyzed using either RT- or cryo-SEM of cryo-fractured membrane cross-sections. Since these organic polymer-based membranes are composed of $>85 \%$ liquid, the PA-doped PBI membranes are prone to dehydration and beam damage during analysis in the SEM. Biological specimen also suffers from dehydration and structural collapse during imaging, which is prevented by using cryo-SEM.[3] Typical RT- and cryo-SEM (Hitachi S4700) crosssection images are compared in Fig. 1. The characteristic $3 \mu \mathrm{m}$ spheroidal features observed in the cryo-SEM image (Fig. 1a) are distorted to $1.7 \mu \mathrm{m}$ when observed at RT. Increased temperature, beam damage, and vacuum exposure cause sublimation, which removes volatile substances from the surface and reveals the fine structure within the membrane. However, care must be taken to avoid over-sublimation. Cryo-SEM imaging of the membrane surface (Fig. 2.) shows spheriodal features, which are faintly evident in the surrounding unetched surface and exposed with beam etching for short times. Cryo-SEM surfaces (Fig. 3A) demonstrate progressive particle sintering with increased vacuum and beam exposure. The fine-scale gel morphology shown in Fig. 3B is composed of $\sim 65 \mathrm{~nm}$ diameter domains with $\sim 30 \mathrm{~nm}$ walls. The beam-sensitivity of cryo-fractured PA-doped PBI membranes prevented energy dispersive X-ray spectroscopy (EDS) analysis in the SEM. In the future, the PA-doped PBI membranes will be cryo-microtomed and cryo-transferred for elemental analysis in a transmission electron microscope (TEM).[4]

References

[1] S. Yu et al. Fuel Cells, 8 (2008) 165. and K. A. Perry et al. J Power Sources 177 (2008) 478.

[2] L. Xiao et al. Chem. Mater. 17 (2005) 5328.

[3] S. L. Erlandsen. in Biological Low-Voltage Scanning Electron Microscopy, Eds. H. Schatten and J. B. Pawley, Springer, New York, 2008, pp. 215-228. 
[4] Research at the Oak Ridge National Laboratory SHaRE User Facility was sponsored by the Scientific User Facilities Division, Office of Basic Energy Sciences, U.S. Department of

1A Energy. Part of this work was supported by HFCIT, NSF-IGERT, and HERE at ORNL.

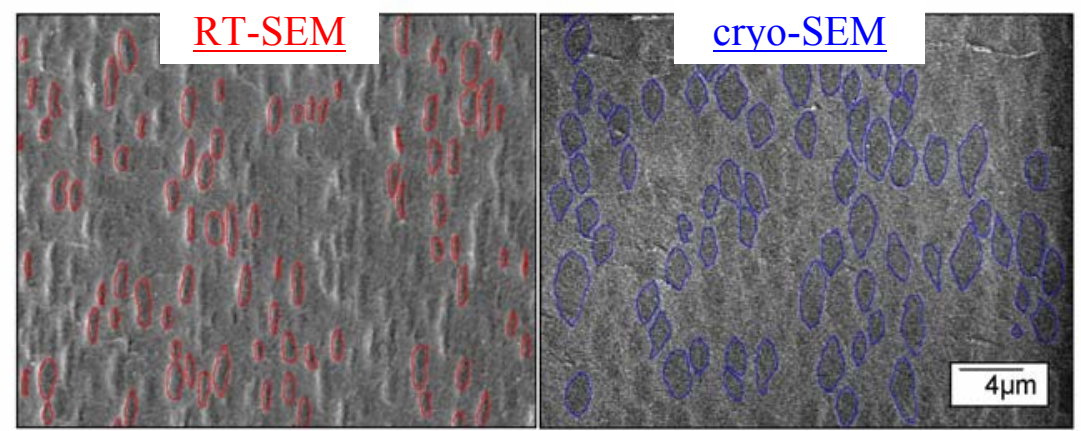

FIG. 1A. Cryo-fracture cross-sections of PA-doped paraPBI membranes imaged with two different SEM techniques (1) room temperature (RT) coated with Ir and (2) uncoated cryo-prepared with no heat induced sublimation imaged at $-157^{\circ} \mathrm{C}$. 1B. The length and breadth of the particles reduces under RT-SEM conditions.

2

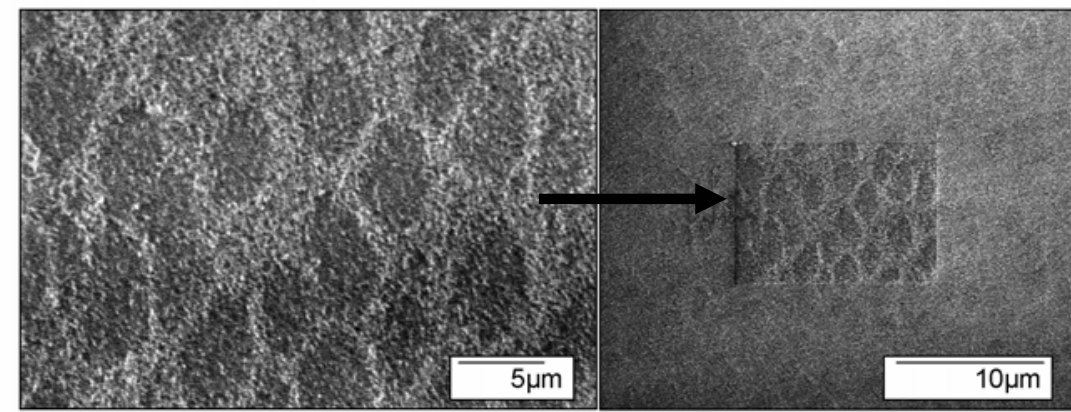

FIG. 2. Cryo-SEM image of PA-doped para-PBI membranes exhibiting spheroidal particles that are highlighted by beam etching.

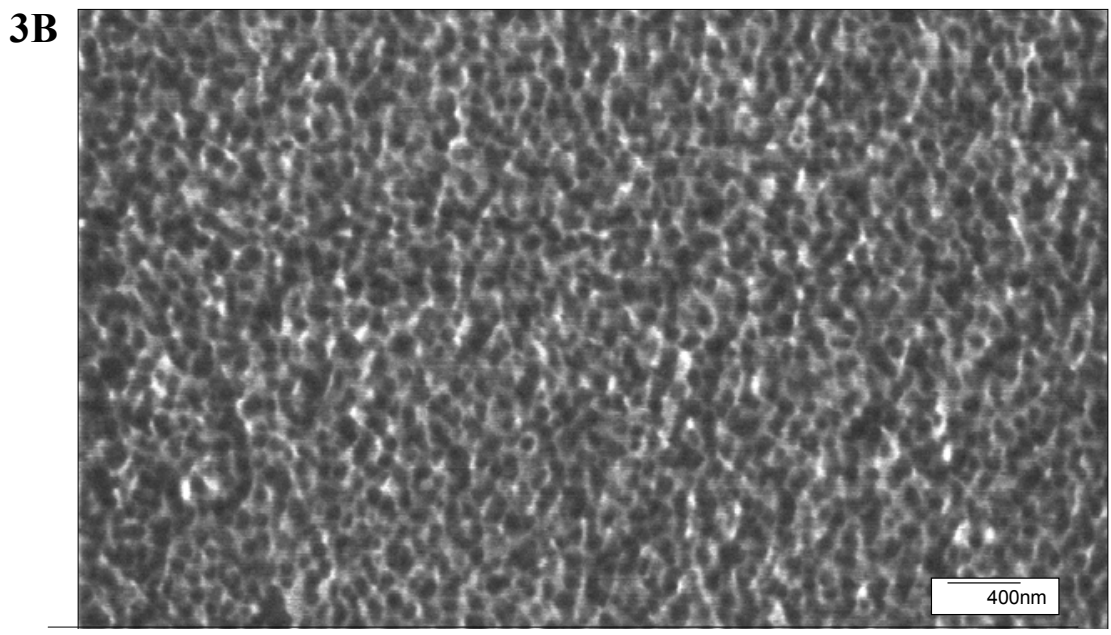

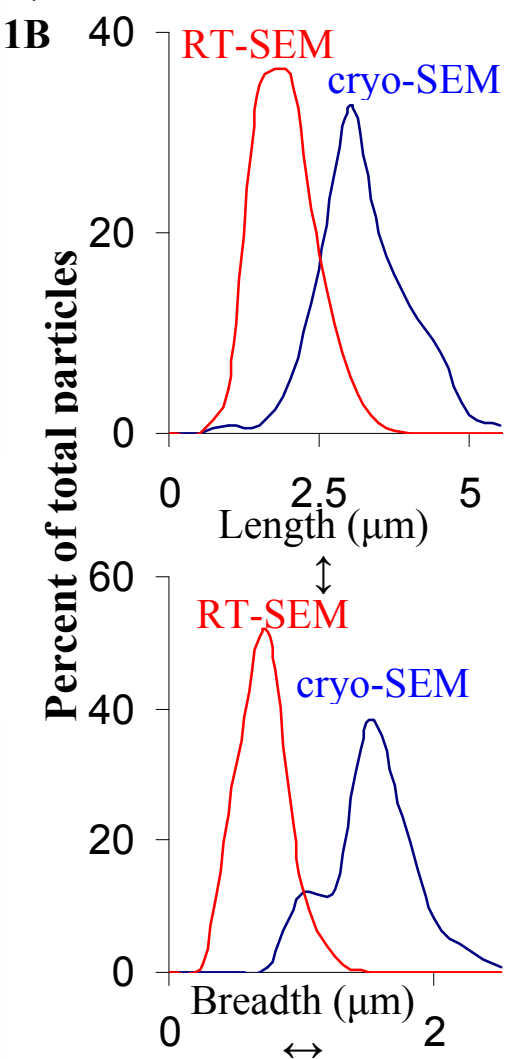

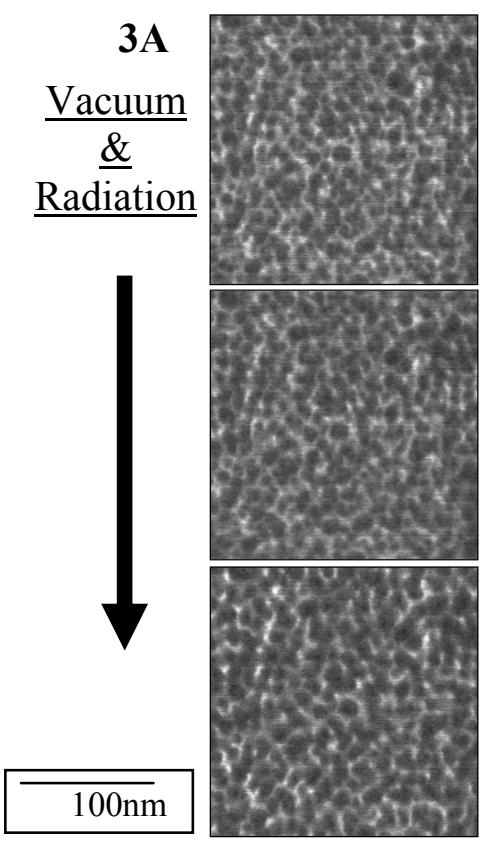

FIG 3A. Sequential cryo-SEM images of uncoated membrane that underwent particle sintering from the combined effect of radiation exposure and vacuum dehydration. 3B. Cryo-SEM image of uncoated PA-doped para-PBI membrane exhibits fine grain structure of $\sim 65 \mathrm{~nm}$ with $\sim 30 \mathrm{~nm}$ walls. 